\title{
Abstracts Presented at the International Neuropsychological Society, British Neuropsychological Society and the Division of Neuropsychology of the British Psychological Society Joint Mid-Year Meeting
}

\author{
July 6-9, 2005
}

Dublin, Ireland

academic achievement . . . . . . . . . . . . . 79 activities of daily living / adaptive functioning . . . 5, 47, 51,

. . . . . . . . 64, 80

ADHD . . . . . . . . . . . . . . . . 43, 71

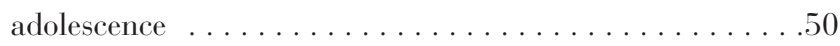

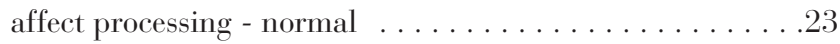

affective processing disorders . . . . . . . 4, 21, 24

aging - normal $\ldots \ldots \ldots \ldots \ldots, 62,67,75,76$

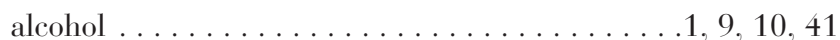

amyotrophic lateral sclerosis $\ldots \ldots \ldots \ldots \ldots \ldots \ldots$

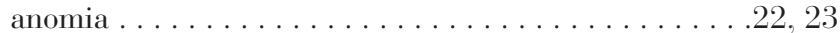

anosognosia $\ldots \ldots \ldots \ldots \ldots \ldots \ldots \ldots \ldots \ldots \ldots \ldots \ldots \ldots, 54,64$

anterior communicating artery aneurysm . . . . . . 17

antisocial behavior ................ . 41

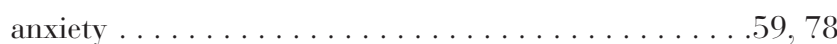

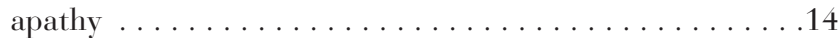

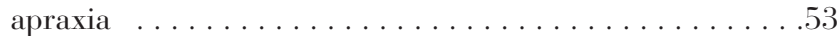

arithmetic ability disorders $\ldots \ldots \ldots \ldots \ldots \ldots \ldots \ldots \ldots$

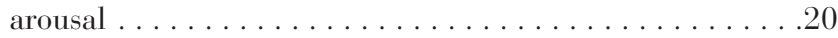

assessment $\ldots .27,36,51,55,57,63,64,68,69,70,80,81$

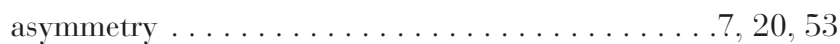

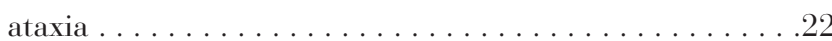

attention .....1, 2, 3, 5, 11, 24, 25, 27, 34, 37, 40, 43, 47, $.54,55,64,67,77$

autism spectrum disorders $\ldots \ldots \ldots \ldots \ldots \ldots \ldots$ awareness .......7, 23, 24, 25, 33, 49, 50, 54, 55, 56

bipolar disorder . . . . . . . . . . . . . . . . . . . . 19

brain damage . . . . . . . . . . . . . . . . . . . . .3.58

brain development $\ldots \ldots \ldots \ldots \ldots \ldots \ldots \ldots \ldots$

brain disorder . . . . . . . . . . . . . . . . . . .42

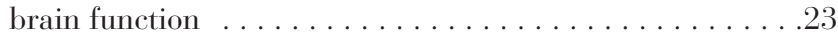

brain injury $49,50,53,54,56,58,67,70,72,73,76,77,81$

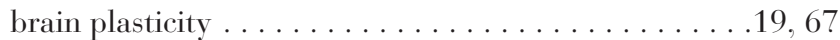

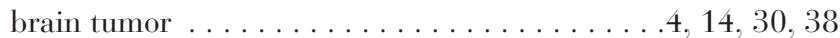

bulimia nervosa . . . . . . . . . . . . . . . . . . . 14 cancer .......................440,79

carbon monoxide . . . . . . . . . . . . . . . . . 18

cardiovascular disease . . . . . . . . . . . . . . . .40

cerebral blood flow . . . . . . . . . . . . . . . . . . 18

cerebrevascular accident/stroke . . . . . . . . .5, 24, 59, 71

cerebrovascular disease . . . . . . . . . . . . . . . .58

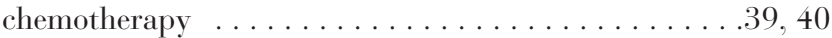

child brain injury . . . .14, 15, 16, 18, 29, 30, 57, 66, 73, 74

child development - abnormal/disease . . .8, 20, 27, 28, 30.

$44,45,73,74,79$

child development - normal . . . . . . . .11, 27, 45, 74

clock drawing . . . . . . . . . . . . . . . . . . . .61

cognitive ......................4. 78

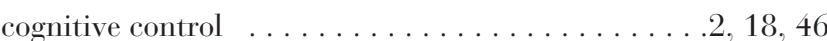

cognitive course . . . . . . . . . . . . . . . . .65

cognitive functioning . . . . .8, 12, 28, 31, 32, 33, 36, 37, 38 , $.39,40,42,46,47,50,51,52,53,60,65,69,70,81$

cognitive neuroscience . . . . . . . 19, 20, 26, 45, 68

cognitive processing . . . . . 19, 40, 49, 50, 52, 57, 68

cognitive rehabilitation . . . . .4, 22, 28, 34, 56, 59, 67, 68, $77,78,79,80$

cognitive reserve / brain reserve capacity . . . . . . . . . 75

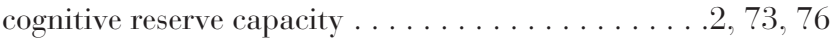

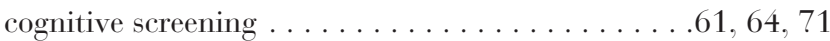

computerized neuropsychological testing . . . 43, 63, 71, 74

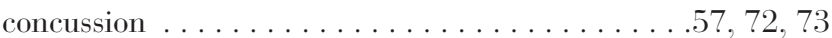

confabulation . . . . . . . . . . . . . . . . . . . 17

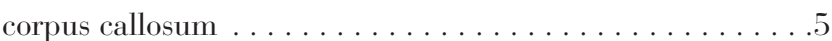

corticobasal degeneration . . . . . . . . . . . . . . .48

creativity . . . . . . . . . . . . . . . . . . . . . 14

cross-cultural . . . . . . . . . . . . . . . . . . . . . . 69

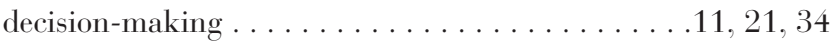

deep brain stimulation . . . . . . . . . . . . . . . .65

dementia - AD ....10, 18, 39, 47, 54, 60, 61, 62, 63, 65,

$69,70,78,80$

dementia - other cortical . . . . . . .35, 47, 61, 64, 80, 81 
demographic effects on test performance

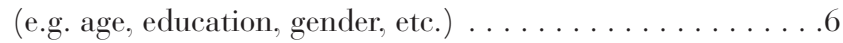
depression ..........24, 33, 34, 35, 36, 50, 59 diabetes . . . . . . . . . . . . . . . . . . . 39

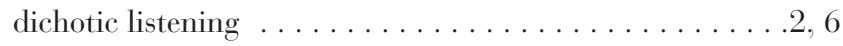

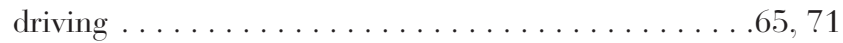
dyslexia ...................... 44

ecological validity $\ldots \ldots \ldots \ldots \ldots$. 46

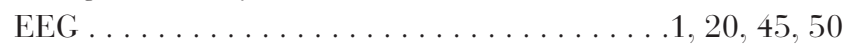

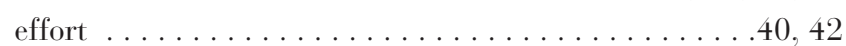
event-related potentials .........7, 19, 20, 23, 53 executive function - abnormal . . . . .12, 13, 14, 22, 33, 36, $\ldots \ldots \ldots \ldots \ldots \ldots \ldots \ldots \ldots \ldots \ldots, 47,59,63,66,75$ executive function - normal $\ldots \ldots \ldots 1,8,27,45,57,67$ executive skills ............28, 34, 58, 76

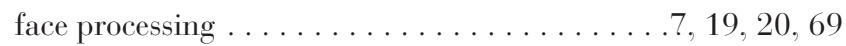

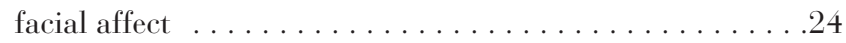

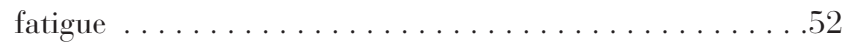

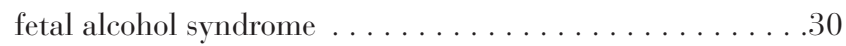
fluency . . . . . . . . . . . . . . . . . . . 10

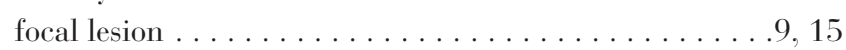
forensic neuropsychology $\ldots \ldots \ldots . \ldots 36,37,38,70$

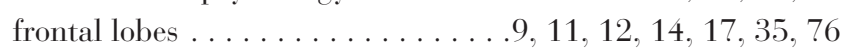
frontal-subcortical circuitory $\ldots \ldots \ldots \ldots \ldots \ldots \ldots$ frontostriatal circuitry . . . . . . . . . . . . . 12 frontotemporal dementia $\ldots \ldots \ldots \ldots \ldots$. . . . . 52 genetic neuropsychology $\ldots \ldots \ldots \ldots$. 4 4 , 65

head injury - closed . . . . . . . . . . . . . . 33

hippocampus ................... . 18

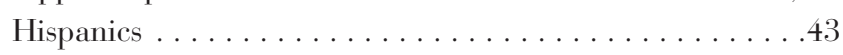

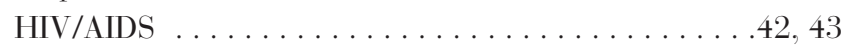

hormone effects ..............42,61

hormone replacement therapy $\ldots \ldots \ldots . \ldots 38,77$

huntington's disease $\ldots \ldots \ldots \ldots \ldots \ldots \ldots \ldots \ldots \ldots$

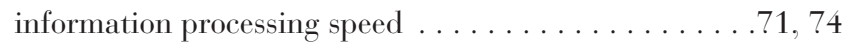

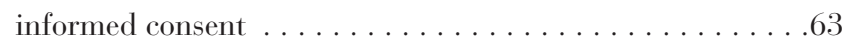

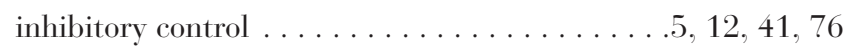
intelligence ..................... 75

language $\ldots \ldots \ldots \ldots \ldots \ldots \ldots \ldots \ldots \ldots \ldots \ldots \ldots \ldots \ldots \ldots \ldots, 22,23,80$ language: aphasia $\ldots \ldots \ldots \ldots, 21,22,48,58,79$ language: development . . . . . . . . . . . . . . 27 laterality ....................6, 11

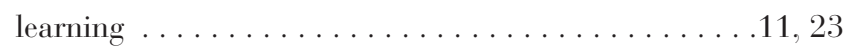

learning disabilities $\ldots \ldots \ldots \ldots \ldots$. . . . . . . . . . . . . 434 low birth weight $\ldots \ldots \ldots \ldots \ldots \ldots \ldots \ldots \ldots \ldots \ldots$, 74

magnetoencephalography . . . . . . . . . . . . . 20 . . . . . . . . . . . .

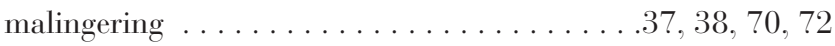

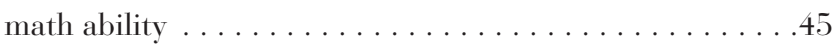
medical illness . . . . . . . . . . . . . . . .40

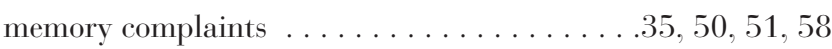
memory disorders . . . . .7, 8, 9, 10, 11, 16, 17, 26, 28, 33, $\ldots \ldots \ldots . \ldots \ldots 4,43,48,58,60,62,66,74,78,81$

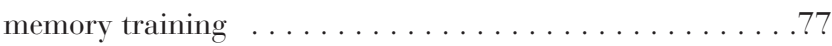

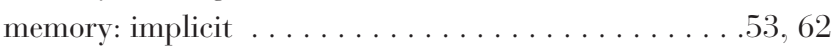
memory: normal .........8, 9, 10, 11, 67, 76 memory: prospective $\ldots \ldots \ldots . \ldots, 56,66,67,76,77$

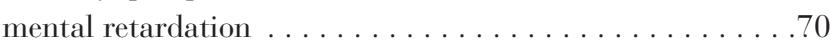

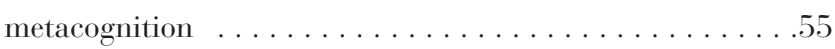

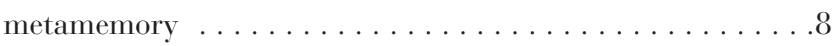
mild cognitive impairment $\ldots \ldots 10,39,43,60,61,63,64$, $\ldots \ldots \ldots \ldots \ldots \ldots \ldots \ldots \ldots \ldots \ldots$ mild traumatic brain injury $\ldots \ldots \ldots . \ldots 29,35,72,73$ motivation . . . . . . . . . . . . . . . . . .17 motor function . . . . . . . 2, 3, 4, 19, 36, 37, 44, 53

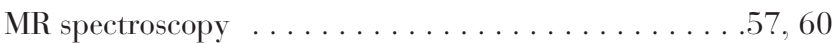
MRI: functional $\ldots \ldots \ldots \ldots \ldots \ldots \ldots \ldots$ 21, 26, 66, 67 MRI: structural .........1, 18, 19, 28, 57, 59, 73 multiple sclerosis $\ldots \ldots \ldots \ldots \ldots \ldots \ldots \ldots$. $\ldots \ldots 2,79$

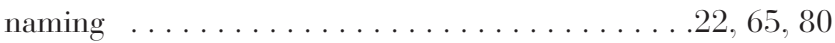

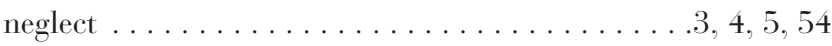

neural circuitry . . . . . . . . . . . . . . . . . . . .29 neurocognition ... . . . . . . . . . . . . . . . . 44 neuroimaging: functional . . . . . . . . . . . . . . . 19 neuroimaging: structural . . . . . . . . . . . 24, 39 neuropsychological assessment . . . 13, 18, 27, 28, 35, 37, . . 38, 39, 41, 42, 43, 46, 47, 58, 59, 61, 64, 66, 70, 71, 72 neuropsychological outcome . . 13, 29, 30, 39, 41, 51, 59, 68

outcome ........32, 34, 52, 56, 57, 58, 60, 76, 81

P300 . . . . . . . . . . . . . . . . . .79

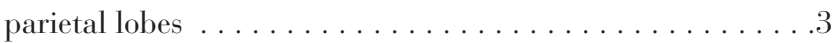

parkinson's disease ............. 53, 64, 65, 66

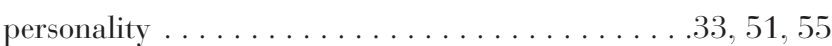

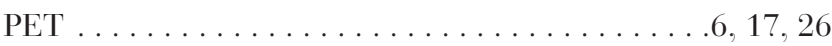

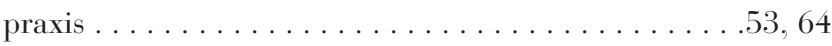

premorbidfunctioning $\ldots \ldots \ldots \ldots \ldots \ldots \ldots, 81$ preterm children $\ldots \ldots \ldots \ldots \ldots, 28,31,32$ proprioception $\ldots \ldots \ldots \ldots \ldots \ldots \ldots \ldots \ldots$

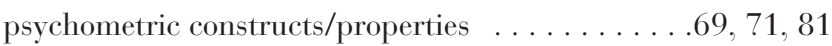
psychopathology: other $\ldots \ldots \ldots . \ldots 34,35,36,70$ 
psychopathy $\ldots \ldots \ldots \ldots \ldots \ldots \ldots \ldots \ldots \ldots \ldots \ldots \ldots$

psychopharmacology . . . . . . . . . . . . . . . . 29

PTSD . . . . . . . . . . . . . . . . . . . . . . .29

quality of life

$.50,51,58,60,66$

reaction time $\ldots \ldots \ldots \ldots \ldots \ldots \ldots \ldots \ldots \ldots \ldots \ldots \ldots \ldots$

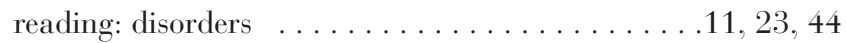

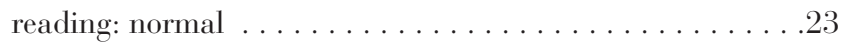

retrosplenial cortex $\ldots \ldots \ldots \ldots \ldots \ldots \ldots \ldots$

schizophrenia ............ 12, 36, 37, 71, 80

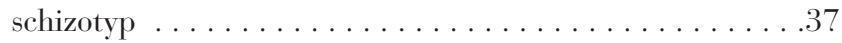

seizure disorders / epilepsy . . . . . . . . . . 26, 50

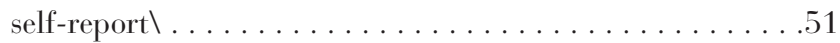

semantic processing ..........9, 47, 80, 81

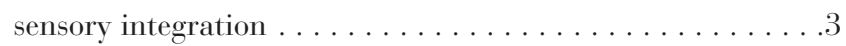

sleep ..................... 77

sleep disorders . . . . . . . . . . . . . . . . 28

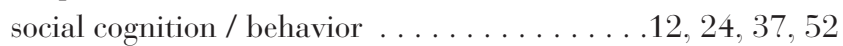

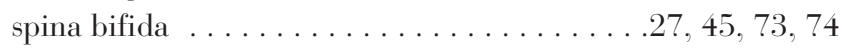

sports-related neuropsychology . . . . . . . . . . 57

stimulants . . . . . . . . . . . . . . . . .79 stroke recovery $\ldots \ldots \ldots \ldots \ldots \ldots \ldots, 21,24,59,60$

subarachnoid hemorrhage . . . . . . . . . . . . . 58

subcortical . . . . . . . . . . . . . . . . . . . . 19

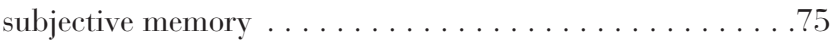

substance abuse $\ldots \ldots \ldots \ldots \ldots \ldots \ldots \ldots \ldots, 41,4 \ldots \ldots$

temporal lobe . . . . . . . . . . . . . . . . 17

test development $\ldots \ldots \ldots \ldots \ldots \ldots \ldots \ldots \ldots \ldots .71$

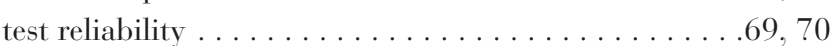

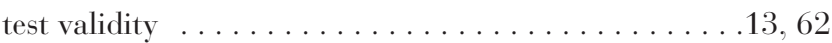

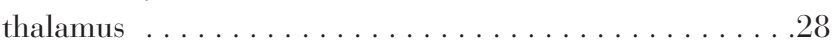

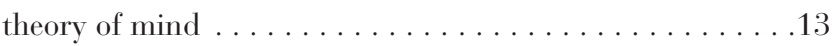

traumatic brain injury . . . . . . 7, 12, 13, 16, 18, 24, 32, 33, $\ldots \ldots 36,37,42,46,49,55,56,57,66,68,72,74,78,79$ treatment outcome ............24, 25, 77, 79

vascular dementia . . . . . . . . . . . . . . . . . 18

verbal abilities . . . . . . . . . . . . . . . . .63,69

visuoconstruction . . . . . . . . . . . . . . . 62

visuospatial $\ldots \ldots \ldots \ldots \ldots \ldots \ldots \ldots \ldots \ldots \ldots \ldots, 23,27$

working memory .1, 2, 3, 4, 7, 10, 11, 20, 21, 23, 33, 63, 65 writing $\ldots \ldots \ldots \ldots \ldots \ldots \ldots \ldots \ldots \ldots \ldots \ldots \ldots$ 